\title{
Diffraction and quasiclassical limit of the Aharonov-Bohm effect
}

\author{
Yu.A. Sitenko ${ }^{1}$ and N.D. Vlasii ${ }^{1,2}$ \\ ${ }^{1}$ Bogolyubov Institute for Theoretical Physics, \\ National Academy of Sciences, 03680, Kyiv, Ukraine \\ ${ }^{2}$ Physics Department, Taras Shevchenko National University of Kyiv, \\ 01601, Kyiv, Ukraine
}

\begin{abstract}
Since the Aharonov-Bohm effect is the purely quantum effect that has no analogues in classical physics, its persistence in the quasiclassical limit seems to be hardly possible. Nevertheless, we show that the scattering Aharonov-Bohm effect does persist in the quasiclassical limit owing to the diffraction, i.e. the Fraunhöfer diffraction in the case when space outside the enclosed magnetic flux is Euclidean, and the Fresnel diffraction in the case when the outer space is conical. Hence, the enclosed magnetic flux can serve as a gate for the propagation of short-wavelength, almost classical, particles. In the case of conical space, this quasiclassical effect which is in principle detectable depends on the particle spin.
\end{abstract}

PACS: 03.65.Nk, 03.65.Vf, 72.80.Vp, 98.80.Cq

Keywords: magnetic vortex, conical space, quantum-mechanical scattering

\section{Introduction}

The Aharonov-Bohm (AB) effect [1] plays a fundamental role in modern physics. It demonstrates that quantum matter is influenced by electromagnetic field even in the case when the region of nonvanishing field strength does not overlap with the region accessible to quantum matter; the indispensable condition is that the latter region be non-simply-connected. A particular example is a magnetic field of 
an infinitely long solenoid which is shielded and made impenetrable to quantum matter; such a field configuration may be denoted as an impenetrable magnetic vortex. Although nowadays the $\mathrm{AB}$ effect is generalized in various aspects and in different areas of modern physics, in the present letter we shall discuss its traditional formulation as of a quantum-mechanical scattering effect off an impenetrable magnetic vortex (see reviews in [2, 3, 4, 5, 6]). Even in this restricted sense, it corresponds to two somewhat different but closely related setups. The first one concerns the fringe shift in the interference pattern due to two coherent particle beams under the influence of an impenetrable magnetic vortex placed between the beams. The second one deals with scattering of a particle beam directly on an impenetrable magnetic vortex. Almost all experiments are performed in the first setup, though the second setup is more elaborate from the theoretical point of view. A direct scattering experiment involving long-wavelength (slow-moving) particles is hardly possible, but that involving short-wavelength (fast-moving) particles is quite feasible, and in the present letter we propose to perform such an experiment.

In classical theory, scattering off an impenetrable magnetic vortex is independent of the vortex flux, as well as of the energy of a scattered particle. Quantummechanical scattering depends on the scattered-particle energy (or wavelength). Although the $\mathrm{AB}$ phase which is acquired by encircling the vortex and related to its flux is independent of the particle wavelength, the question is how to observe this phase for different wavelengths and for different scattering angles. The differential cross section in the limit of long (as compared to the vortex thickness) wavelengths was shown to depend periodically on the vortex flux for all scattering angles [1], and namely this (the periodic dependence on the enclosed flux) is generically referred to as the AB effect, see, e.g., [3]. The long-wavelength limit corresponds to the ultraquantum limit when the wave aspects of matter are exposed to the maximal extent.

As the particle wavelength decreases, the wave aspects of matter are suppressed in favour of the corpuscular ones, and therefore the persistence of the $\mathrm{AB}$ effect in the limit of short (as compared to the vortex thickness) wavelengths seems to be rather questionable. Actually, there is a controversy in the literature concerning this point. As it follows from [2, 3], scattering off an impenetrable magnetic vortex in the short-wavelength limit tends to classical scattering which is independent of the vortex flux, and thus the AB effect is extinct in this limit. On the other hand, it was already shown by Aharonov and Bohm 1] for the case of an idealized (infinitely thin) vortex that the wave function vanishes in the strictly forward direction, when the vortex flux equals a half-of-odd-integer multiple of the London flux quantum; later this result was generalized to the case of a realistic vortex of finite thickness [5] and, being independent of the value of the particle wavelength, it persists in the short-wavelength limit. Thus, this 
circumstance witnesses in favour of the persistence of the $\mathrm{AB}$ effect, since the wave function for all other values of the vortex flux is for sure nonvanishing in the forward direction.

The exclusiveness of the forward direction is of no surprise. We recall the wellknown fact that the short-wavelength limit of quantum-mechanical scattering off a hard core does not converge with the classical point-particle scattering (perfect reflection), it differs by a forward peak which is due to the Fraunhöfer diffraction; the peak is increasing, as the particle wavelength is decreasing and the particle is becoming like a classical point corpuscle. Meanwhile the width of the forward peak is decreasing, and that is why the experimental detection of the peak is a rather hard task. As is noted in [7, it seems more likely that the measurable quantity is the classical cross section, although the details of this phenomenon depend on the method of measurement. On the other hand, the forward peak cannot in any way be simply ignored, because its amplitude is involved in the optical theorem, whereas the amplitude yielding classical scattering vanishes in the forward direction. Also, the forward peak contributes considerably to the total cross section, making the latter twice as large as the classical total cross section.

Thus, the quantum-mechanical scattering effects persist in the quasiclassical limit owing to the diffraction effects persisting in the short-wavelength limit. Concerning the AB effect, this conjecture is justified quantitatively in the present letter.

However, our consideration is focused mainly on the scattering AB effect in conical space. Conical space is a space which is locally flat almost everywhere with exception of a region in the form of an infinitely long tube; the metric outside the tube is given by squared length element [8, 9, 10]

$$
\mathrm{d} s^{2}=(1-\eta)^{-2} \mathrm{~d} \tilde{r}^{2}+\tilde{r}^{2} \mathrm{~d} \varphi^{2}+\mathrm{d} z^{2}=\mathrm{d} r^{2}+r^{2} \mathrm{~d} \tilde{\varphi}^{2}+\mathrm{d} z^{2},
$$

where

$$
\tilde{r}=r(1-\eta), \quad 0<\varphi<2 \pi, \quad 0<\tilde{\varphi}<2 \pi(1-\eta),
$$

and $\eta$ is related to the curvature integrated over the transverse section of the tube, being of the same sign. Deficit angle $2 \pi \eta$ is bounded from above by $2 \pi$ and is unbounded from below (quantity $-2 \pi \eta$ for negative $\eta$ is the proficit angle that can be arbitrarily large), thus $-\infty<\eta<1$. Conical space emerges inevitably as an outer space of a topological defect in the form of a string; such defects known as the Abrikosov-Nielsen-Olesen vortices [11, 12] arise as a consequence of phase transitions with spontaneous breakdown of gauge symmetries, when the first homotopy group of the group space of the broken symmetry group is nontrivial. Certainly, the value of $\eta$ is vanishingly small for vortices in superconductors, but vortices under the name of cosmic strings [13, 14] are currently 
discussed in cosmology and astrophysics, and the observational data is consistent with the values of $\eta$ in the range $0<\eta<4 \cdot 10^{-7}$ (see, e.g., [15]), although the direct evidence for the existence of cosmic strings is still lacking. In carbon nanophysics, topological defects in graphene (two-dimensional crystal of carbon atoms) correspond to nanocones with the values of $\eta$ equal to positive and negative multiples of $1 / 6$ [16, 17]. At last, conical space may emerge in a rather general context of contemporary condensed matter physics which operates with a variety of two-dimensional structures (thin films) made of different materials. If such a film is rolled into a cone, then one can generate quasiparticle excitations in this conically-shaped film and consider their propagation towards and through the tip. In all above setups, the problem of quantum-mechanical scattering of a nonrelativistic particle by a magnetic vortex in conical space may be relevant.

Scattering in an idealized (with the core of zero transverse size) conical space was considered by 't Hooft [18] and Jackiw et al [19, 20]; later the consideration was extended to the case of an idealized magnetic vortex placed along the axis of an idealized conical space [21]. However, in the quasiclassical limit the effects of nonzero transverse size of the core become important. These effects were taken properly into account in [22] (see also [23, 24]), and we shall implicate the results of the latter works. Instead of using the quasiclassical WKB approximation or an analogue of the Kirchhoff approximation in optics, we shall get the quasiclassical limit directly from exact expressions for the scattering amplitude.

\section{Quantum-mechanical scattering off an impen- etrable magnetic vortex}

A scattering wave solution to the Schrödinger equation $\mathrm{i} \hbar \partial_{t} \psi=H \psi$ in the case of a cylindrically symmetric potential has the following asymptotics at large distances from the symmetry axis:

$$
\psi \sim \exp \left(-\mathrm{i} E t \hbar^{-1}+\mathrm{i} k_{z} z\right)\left[\psi_{\text {in }}(\mathbf{r} ; \mathbf{k})+f(k, \varphi) \exp (\mathrm{i} k r) \sqrt{\frac{\mathrm{i}}{r}}+O\left(r^{-1}\right)\right],
$$

where $\mathbf{k}$ is the two-dimensional wave vector which is orthogonal to the symmetry axis, $k^{2}=2 m E \hbar^{-2}-k_{z}^{2}>0, \varphi$ is the angle between vectors $\mathbf{r}$ and $\mathbf{k}, m$ and $E$ are the mass and the energy of a scattered particle. Meantime, in the framework of the time-dependent scattering theory, one gets $S$-matrix:

$S\left(k, \varphi, k_{z} ; k^{\prime}, \varphi^{\prime}, k_{z}^{\prime}\right)=\delta\left(k_{z}-k_{z}^{\prime}\right)\left[I\left(k, \varphi ; k^{\prime}, \varphi^{\prime}\right)+\delta\left(k-k^{\prime}\right) \frac{\mathrm{i}}{\sqrt{2 \pi k}} f\left(k, \varphi-\varphi^{\prime}\right)\right]$, 
where the final $(\mathbf{k})$ and initial $\left(\mathbf{k}^{\prime}\right)$ two-dimensional wave vectors are written in polar variables; $f(k, \varphi)$ in $(2)$ and $(3)$ is the scattering amplitude. In the case of the short-range interaction, the first term in square brackets in (3) is the unity matrix, $I\left(k, \varphi ; k^{\prime}, \varphi^{\prime}\right)=\delta\left(k-k^{\prime}\right) k^{-1} \Delta\left(\varphi-\varphi^{\prime}\right)$, where $\Delta(\varphi)=(2 \pi)^{-1} \sum_{n \in \mathbb{Z}} e^{\mathrm{i} n \varphi}$ is the delta-function for a compact (angular) variable, $\Delta(\varphi+2 \pi)=\Delta(\varphi), \mathbb{Z}$ is the set of integer numbers. Appropriately, the incident wave (first term in square brackets in (2)) is a plane wave, $\psi_{\text {in }}(\mathbf{r} ; \mathbf{k})=\exp (\mathrm{i} k r \cos \varphi)$, and the optical theorem expressing the probability conservation takes form $2 \sqrt{\frac{2 \pi}{k}} \operatorname{Im} f(k, 0)=$ $\sigma_{\text {tot }}$.

If magnetic flux $\Phi$ is enclosed into an impenetrable tube of radius $r_{c}$, then the particle wave function obeys the Dirichlet boundary condition at the edge of the tube, $\left.\psi\right|_{r=r_{c}}=0$, and the Schrödinger hamiltonian for a spinless nonrelativictic particle takes form

$$
H=-\frac{\hbar^{2}}{2 m}\left[\partial_{r}^{2}+\frac{1}{r} \partial_{r}+\frac{1}{(1-\eta)^{2} r^{2}}\left(\partial_{\varphi}-\mathrm{i} \frac{\Phi}{\Phi_{0}}\right)^{2}+\partial_{z}^{2}\right],
$$

where $\Phi_{0}=2 \pi \hbar c e^{-1}$ is the London flux quantum. The interaction in this case is not of the potential type and is even nondecreasing at large distances from the centre (flux enclosed in the tube).

\subsection{AB effect in Euclidean space}

Already in the case of an impenetrable magnetic vortex in Euclidean space $(\eta=$ 0 ), the long-range nature of interaction leads to a distortion of the unity matrix in (3), which is now given by

$$
I\left(k, \varphi ; k^{\prime}, \varphi^{\prime}\right)=\cos \left(\Phi \Phi_{0}^{-1} \pi\right) \delta\left(k-k^{\prime}\right) k^{-1} \Delta\left(\varphi-\varphi^{\prime}\right) ;
$$

appropriately, the incoming wave in $(2)$ is distorted, and factor $\exp \left[\mathrm{i} \Phi \Phi_{0}^{-1}(\varphi-\pi)\right]$ emerges in addition to the plane wave [1. The scattering amplitude in this case takes form

$$
f(k, \varphi)=f_{0}(k, \varphi)+f_{c}(k, \varphi),
$$

where $f_{0}$ corresponds to the idealized case of the tube of zero transverse size (vortex of zero thickness), whereas all the finite-thickness effects are contained in $f_{c}$. In the long-wavelength limit, $k r_{c} \ll 1$, the finite-thickness effects are negligible, and the scattering amplitude is given by $f_{0}$ which was first obtained in [1] where it was shown to be a periodic function of $\Phi$ with the period equal to $\Phi_{0}$, diverging in the forward direction. The total cross section diverges as well, and, although the probability is certainly conserved, the optical theorem in this limit is hardly informative, being a relation between two divergent quantities. 
On the contrary, in the short-wavelength limit, $k r_{c} \gg 1$, the finite-thickness effects are prevailing, since $f_{0}$ is of order $\sqrt{r_{c}} O\left[\left(k r_{c}\right)^{-1 / 2}\right]$, and the scattering amplitude is given by

$$
f_{c}(k, \varphi)=f^{(\text {class })}(k, \varphi)+f^{(\text {peak })}(k, \varphi)+\sqrt{r_{c}} O\left[\left(k r_{c}\right)^{-1 / 6}\right],
$$

where $f^{\text {(class) }}$ yields the classical differential cross section:

$$
\frac{\mathrm{d} \sigma^{\text {(class })}}{\mathrm{d} \varphi}=\left|f^{\text {(class) }}(k, \varphi)\right|^{2}=\frac{r_{c}}{2} \sin \frac{\varphi}{2} \quad(0<\varphi<2 \pi),
$$

and $f^{\text {(peak) }}$ yields the differential cross section of the Fraunhöfer diffraction in the forward $(\varphi=0)$ direction:

$$
\begin{gathered}
\frac{\mathrm{d} \sigma^{\text {(peak })}}{\mathrm{d} \varphi}=\left|f^{(\text {peak })}(k, \varphi)\right|^{2}=2 r_{c}\left\{\cos \left(2 \Phi \Phi_{0}^{-1} \pi\right) \Delta_{k r_{c}}(\varphi)\right. \\
\left.+\left[1-\cos \left(2 \Phi \Phi_{0}^{-1} \pi\right)-\sin \left(2 \Phi \Phi_{0}^{-1} \pi\right) \sin \left(k r_{c} \varphi\right)\right] \Delta_{\frac{1}{2} k r_{c}}(\varphi)\right\} .
\end{gathered}
$$

Here $\Delta_{x}(\varphi)$ is a regularized (smoothed) delta-function for the angular variable, which is defined for $-\pi<\varphi<\pi$ by relations

$$
\lim _{x \rightarrow \infty} \Delta_{x}(\varphi)=\Delta(\varphi), \quad \Delta_{x}(0)=\frac{x}{\pi},
$$

and, hence, (9) is strongly peaked in the strictly forward direction:

$$
\begin{array}{r}
\frac{\mathrm{d} \sigma^{(\text {peak })}}{\mathrm{d} \varphi}=\frac{2}{\pi} k r_{c}^{2}\left\{\cos ^{2}\left(\Phi \Phi_{0}^{-1} \pi\right)-\frac{1}{2} k r_{c} \varphi \sin \left(2 \Phi \Phi_{0}^{-1} \pi\right)\right. \\
\left.-\frac{1}{24}\left(k r_{c} \varphi\right)^{2}\left[1+7 \cos \left(2 \Phi \Phi_{0}^{-1} \pi\right)\right]\right\}\left\{1+O\left[\left(k r_{c} \varphi\right)^{2}\right]\right\}, \quad|\varphi| \ll\left(k r_{c}\right)^{-1} .
\end{array}
$$

The optical theorem in the short-wavelength limit takes form

$$
\sin ^{2}\left(\Phi \Phi_{0}^{-1} \pi\right) \frac{2 \pi}{k} \Delta_{2 k r_{c}}(0)+2 \cos \left(\Phi \Phi_{0}^{-1} \pi\right) \sqrt{\frac{2 \pi}{k}} \operatorname{Im} f^{(\text {peak })}(k, 0)=\sigma_{\text {tot }},
$$

where, due to the contribution of the diffraction peak, the total cross section, $\sigma_{\text {tot }}=\sigma^{\text {(class) }}+\sigma^{\text {(peak) }}=4 r_{c}$, is twice as large as the classical total cross section.

\section{$2.2 \quad$ AB effect in conical space}

If space outside the vortex is conical, then the unity matrix in (3) is modified in the following way [22]:

$$
\begin{array}{r}
I\left(k, \varphi ; k^{\prime}, \varphi^{\prime}\right)=\delta\left(k-k^{\prime}\right)(2 k)^{-1} e^{2 \mathrm{i} k\left(r_{c}-\xi_{c}\right)}\left\{\exp \left[\mathrm{i} \Phi \Phi_{0}^{-1}\left(\pi+\omega_{\eta}\right)\right] \Delta\left(\varphi-\varphi^{\prime}-\omega_{\eta}\right)\right. \\
\left.+\exp \left[-\mathrm{i} \Phi \Phi_{0}^{-1}\left(\pi+\omega_{\eta}\right)\right] \Delta\left(\varphi-\varphi^{\prime}+\omega_{\eta}\right)\right\} \\
\end{array}
$$


where $\xi_{c}=\int_{0}^{r_{c}} \mathrm{~d} s$ is the geodesic radius of the vortex core (note that spatial region $r<r_{c}$ is characterized by nonzero curvature), and $\omega_{\eta}=\eta \pi(1-\eta)^{-1}$. The scattering amplitude in the long-wavelength limit, $f_{0}(k, \varphi)$, is a periodic function of $\Phi$ with the period equal to $\Phi_{0}$, diverging in two directions which are symmetric with respect to the forward one, $\varphi= \pm \omega_{\eta}$, see [20, 21, 22]. These two directions are the directions along which the Fraunhöfer diffraction occurs, and the scattering amplitude in the short-wavelength limit, $f_{c}(k, \varphi)$, is given by

$f_{c}(k, \varphi)=f^{(\mathrm{q}-\text { class })}(k, \varphi)+f_{+}^{(\text {peak })}\left(k, \varphi-\omega_{\eta}\right)+f_{-}^{(\text {peak })}\left(k, \varphi+\omega_{\eta}\right)+\sqrt{r_{c}} O\left[\left(k r_{c}\right)^{-1 / 6}\right]$,

where $f_{ \pm}^{(\text {peak })}$ yields the differential cross section of the Fraunhöfer diffraction in one or another direction:

$$
\frac{\mathrm{d} \sigma_{ \pm}^{\text {(peak) }}}{\mathrm{d} \varphi}=r_{c}(1-\eta) \Delta_{\frac{1}{2} k r_{c}(1-\eta)}\left(\varphi \mp \omega_{\eta}\right),
$$

which, unlike the case of Euclidean space, is independent of the vortex flux; note that the interference between the amplitudes of different diffraction peaks is flux dependent, but it is suppressed as $r_{c} O\left[\left(k r_{c}\right)^{-1 / 2}\right]$ in the short-wavelength limit. Before turning to the remaining part, $f^{\text {(q-class) }}$, of the scattering amplitude in the short-wavelength limit, let us dwell on classical scattering off a magnetic vortex in conical space.

If the vortex core is impenetrable to a classical point particle, then its scattering does not depend on the vortex flux and, apart from the perfect reflection from the core, is purely kinematic. For a particle with the impact parameter exceeding the core radius, there is no scattering in coordinates $r, \tilde{\varphi}$ (see (1)), but, going over to angular variable $\varphi$, one gets classical trajectories which after bypassing the vortex either diverge or converge (and intersect). In the case $-\infty<\eta<0$, region $\omega_{\eta}<\varphi<-\omega_{\eta}$ is not accessible to incident particles due to the divergence of trajectories; thus, this region may be denoted as the region of classical shadow. In the case $0<\eta<1 / 2$, region $-\omega_{\eta}<\varphi<\omega_{\eta}$ is accessed by incident particles from both sides of the vortex due to the convergence of trajectories; thus, this region may be denoted as the region of classical double image [8, 14]. As $\eta$ increases from $1 / 2$ to 1 , the cases of shadow and double image change each other successively, for more details see [22].

Returning to quantum-mechanical scattering in the short-wavelength limit, we find that $f^{\text {(q-class) }}$ in the case $-\infty<\eta<0$ is vanishing as $\sqrt{r_{c}} O\left[\left(k r_{c}\right)^{-1 / 6}\right]$ in the shadow region, whereas out of this region it yields the finite differential cross section which is independent of the vortex flux:

$$
\frac{\mathrm{d} \sigma^{(\mathrm{q}-\text { class })}}{\mathrm{d} \varphi}=\frac{1}{2} r_{c}(1-\eta)^{2} \sin \left[\frac{1}{2}(1-\eta) \varphi+\frac{1}{2} \eta \pi\right]
$$


these results are consistent with classical theory.

In the double-image region in the case $0<\eta<1 / 2, f^{\text {(q-class) }}$ consists of two terms:

$$
\begin{aligned}
f^{(\mathrm{q}-\text { class })}(k, \varphi)= & -e^{2 \mathrm{i} k\left(r_{c}-\xi_{c}\right)} \sqrt{\frac{r_{c}}{2 \mathrm{i}}}(1-\eta) \sum_{ \pm} \sqrt{\cos \left[\frac{1}{2}(1-\eta)(\varphi \mp \pi)\right]} \times \\
& \times \exp \left\{\mathrm{i} \Phi \Phi_{0}^{-1}(\varphi \mp \pi)-2 \mathrm{i} k r_{c} \cos \left[\frac{1}{2}(1-\eta)(\varphi \mp \pi)\right]\right\} ;
\end{aligned}
$$

the incident wave in this region consists of two terms as well:

$$
\psi_{\text {in }}(\mathbf{x} ; \mathbf{k})=(1-\eta) \sum_{ \pm} \exp \{-\mathrm{i} k r \cos [(1-\eta)(\varphi \mp \pi)]\} \exp \left[\mathrm{i} \Phi \Phi_{0}^{-1}(\varphi \mp \pi)\right] \text {. }
$$

Out of the double-image region, the incident wave is given by one term, as well as is $f^{\text {(q-class) }}$ which yields the differential cross section in the form of (16). Although the case $1 / 2<\eta<1$ can be considered properly, see [22], we shall restrict ourselves to the case $0<\eta<1 / 2$ in the following.

Due to the interference between two terms, the differential cross section corresponding to (17) is dependent on the vortex flux with the period equal to the London flux quantum:

$$
\begin{array}{r}
\frac{\mathrm{d} \sigma^{(\mathrm{q}-\text { class })}}{\mathrm{d} \varphi}=r_{\mathrm{c}}(1-\eta)^{2}\left\{\cos \left(\frac{1}{2}(1-\eta) \varphi\right) \sin \left(\frac{1}{2} \eta \pi\right)+\right. \\
\left.+\sqrt{\sin ^{2}\left(\frac{1}{2} \eta \pi\right)-\sin ^{2}\left(\frac{1}{2}(1-\eta) \varphi\right)} \cos \left[2 \Phi \Phi_{0}^{-1} \pi+4 k r_{\mathrm{c}} \sin \left(\frac{1}{2}(1-\eta) \varphi\right) \cos \left(\frac{1}{2} \eta \pi\right)\right]\right\}, \\
|\varphi|<\omega_{\eta \cdot(19)}
\end{array}
$$

In the forward direction in the case $\sin \left(\frac{1}{2 k r_{c}}\right) \ll \sin \left(\frac{1}{2} \eta \pi\right)$, we get

$$
\begin{array}{r}
\frac{\mathrm{d} \sigma^{(\mathrm{q}-\text { class })}}{\mathrm{d} \varphi}=2 r_{\mathrm{c}}(1-\eta)^{2} \sin \left(\frac{1}{2} \eta \pi\right) \cos ^{2}\left[\Phi \Phi_{0}^{-1} \pi+k r_{c}(1-\eta) \varphi \cos \left(\frac{1}{2} \eta \pi\right)\right] \\
(1-\eta)|\varphi|<\left(k r_{c}\right)^{-1}
\end{array}
$$

The physical reason of the persistence of the $\mathrm{AB}$ effect in the short-wavelength (quasiclassical) limit in the case $0<\eta<1 / 2$ is the Fresnel diffraction that is the diffraction in converging rays, whereas the Fraunhöfer diffraction is the diffraction in almost parallel rays.

The dependence on the vortex flux is washed off after integration over the whole range of the scattering angle, and we get the total cross section in the quasiclassical limit,

$$
\sigma_{\text {tot }}=\sigma^{(\mathrm{q}-\text { class })}+\sigma_{+}^{(\text {peak })}+\sigma_{-}^{(\text {peak })}=4 r_{c}(1-\eta),
$$


which is twice the classical total cross section; the last result is valid for an arbitrary conical space, $-\infty<\eta<1$. The optical theorem takes form

$$
\begin{array}{r}
\cos \left[\Phi \Phi_{0}^{-1}\left(\pi+\omega_{\eta}\right)\right] \sqrt{\frac{2 \pi}{k}} \operatorname{Im}\left\{\left[f_{+}^{(\text {peak })}(k, 0)+f_{-}^{(\text {peak })}(k, 0)\right] e^{-2 \mathrm{i} k\left(r_{c}-\xi_{c}\right)}\right\}- \\
-\sin \left[\Phi \Phi_{0}^{-1}\left(\pi+\omega_{\eta}\right)\right] \sqrt{\frac{2 \pi}{k}} \operatorname{Re}\left\{\left[f_{+}^{(\text {peak })}(k, 0)-f_{-}^{(\text {peak })}(k, 0)\right] e^{-2 \mathrm{i} k\left(r_{c}-\xi_{c}\right)}\right\}+ \\
+\frac{\pi}{k} \Delta_{2 k r_{c}(1-\eta)}(0)=\sigma_{\text {tot }}
\end{array}
$$

\section{Discussion of results and conclusion}

Thus, we can summarize that the scattering AB effect persists in the quasiclassical limit owing to the diffraction. Although the effect is invisible for the cross section integrated over the whole range of the scattering angle, the effect reveals itself for the differential cross section. In the case of a magnetic vortex in Euclidean space, the persistence of the AB effect is due to the Fraunhöfer diffraction which is peaked in the forward direction, see (9) and (11). In the case of a magnetic vortex in conical space, the peak of the Fraunhöfer diffraction is shifted from the forward direction and splitted into two peaks in directions which are symmetric

with respect to the forward one; the contribution of each peak is independent of the vortex flux, see (15). If the forward region between two Fraunhöfer-diffraction peaks is the region of the classical shadow, then the AB effect disappears in the quasiclassical limit. If the forward region between two Fraunhöfer-diffraction peaks is the region of the classical double image, then the persistence of the AB effect in the quasiclassical limit is due to the Fresnel diffraction in this region, see (19) and (20) for the case $0<\eta<1 / 2$.

Since a peak of the Fraunhöfer diffraction is elusive to experimental measurements, it might be hard to detect the vortex flux dependence in the strictly forward direction in Euclidean space. On the contrary, the vortex flux dependence which is due to the Fresnel diffraction in conical space looks much more likely to be detectable: it is spread over the wider region in the forward direction and its amount is finite in the quasiclassical limit, compare (20) with (11). It should be noted that the optical theorem in conical space imposes no restrictions on the scattering amplitude in the strictly forward direction; instead, it involves the scattering amplitude in the strict directions of the Fraunhöfer-diffraction peaks, see $(22)$.

Another distinction of scattering in conical space is that it depends on spin of a scattered particle: the appropriate spin connection which is dependent on $\eta$ should be introduced in hamiltonian (4). In particular, for a spin- $1 / 2$ particle the results are modified in the following way (see [22]): one should change $\Phi \Phi_{0}^{-1}$ 
to $\Phi \Phi_{0}^{-1} \mp \frac{1}{2} \eta$, where two signs correspond to two spin states which are defined by projections of spin on the vortex axis.

If a magnetic vortex is trapped inside a superconducting shel1 1 , then its flux is quantized in the units of a semifluxon, i.e. half of the London flux quantum. In view of (20) we get the following relation in the quasiclassical limit when condition $\sin \left(\frac{1}{2 k r_{c}}\right) \ll \sin \left(\frac{1}{2} \eta \pi\right)$ is satisfied:

$$
\left.\frac{\mathrm{d} \sigma^{\text {(q-class })}}{\mathrm{d} \varphi}\right|_{\Phi=n \Phi_{0}}+\left.\frac{\mathrm{d} \sigma^{\text {(q-class })}}{\mathrm{d} \varphi}\right|_{\Phi=\left(n+\frac{1}{2}\right) \Phi_{0}}=\left.2 \frac{\mathrm{d} \sigma^{\text {(class })}}{\mathrm{d} \varphi}\right|_{\varphi=0}, \quad(1-\eta)|\varphi|<\left(k r_{c}\right)^{-1}
$$

where on the right hand-side stands the doubled differential cross section for the strictly forward scattering of a classical point particle by a hard core. Hence, the quasiclassical limit of the $\mathrm{AB}$ effect in the case when the vortex flux equals an integer multiple of a semifluxon can be presented in the form

$$
\left.\frac{\mathrm{d} \sigma^{\text {(q-class })}}{\mathrm{d} \varphi}\right|_{\Phi=n \Phi_{0} / 2}=\left.F(\varphi, \pm) \frac{\mathrm{d} \sigma^{\text {(class) }}}{\mathrm{d} \varphi}\right|_{\varphi=0}, \quad(1-\eta)|\varphi|<\left(k r_{c}\right)^{-1}
$$

where the upper (lower) sign in $F$ corresponds to even (odd) $n$, and

$$
F(\varphi, \pm)=1 \pm \cos \left[2 k r_{c}(1-\eta) \varphi \cos \left(\frac{1}{2} \eta \pi\right)\right]
$$

for a spinless particle,

$$
F(\varphi, \pm)=1 \pm \cos \left[2 k r_{c}(1-\eta) \varphi \cos \left(\frac{1}{2} \eta \pi\right)\right] \cos (\eta \pi)
$$

for an unpolarized spin-1/2 particle,

$$
F(\varphi, \pm)=1 \pm \cos \left[2 k r_{c}(1-\eta) \varphi \cos \left(\frac{1}{2} \eta \pi\right)-\sigma \eta \pi\right]
$$

for a polarized spin-1/2 particle ( $\sigma= \pm 1$ correspond to two polarization states). We conclude that the enclosed magnetic flux can serve as a gate for shortwavelength, almost classical, particles propagating in conical space; the effect depends on the particle spin, and the most efficient gate is for the propagation of spinless particles in the strictly forward direction, see (25) at $\varphi=0$. For instance, the propagation of fast-moving electronic excitations in the bilayer graphene sample 2 of conical shape can be governed by the magnetic flux applied through a hole at the tip.

\footnotetext{
${ }^{1}$ Since no magnetic field can leak outside, the superconducting shell guarantees for certain that there is no overlap between the region of magnetic flux and the region which is accessible to the scattered particle, see [4, 6].

2 The relevance of hamiltonian (4) rather than the Dirac hamiltonian is due to the quadratic dispersion law in this case, see [25].
} 


\section{Acknowledgments}

The work was partially supported by the Department of Physics and Astronomy

of the National Academy of Sciences of Ukraine under special program "Fundamental properties of physical systems in extremal conditions".

\section{References}

[1] Y. Aharonov, D. Bohm, Phys. Rev. 115, 485 (1959).

[2] S. N. M. Ruijsenaars, Ann. Phys. (NY) 146, 1 (1983).

[3] S. Olariu, I. I. Popescu, Rev. Mod. Phys. 57, 339 (1985).

[4] M. Peshkin, A. Tonomura, The Aharonov-Bohm Effect (Springer-Verlag, Berlin, 1989).

[5] G. N. Afanas'ev, Fiz. Elem. Chastits At. Yadra 21, 172 (Sov. J. Part. Nucl. 21, 74) (1990).

[6] A. Tonomura, J. Phys. A: Math. Theor. 43, 354021 (2010).

[7] P. M. Morse, H. Feshbach, Methods of Theoretical Physics II (McGraw-Hill, New York, 1953) Chapter 11, section 11.2.

[8] L. Marder, Proc. Roy. Soc. London A 252, 45 (1959).

[9] W. Israel, Phys. Rev. D 15935 (1977).

[10] D. D. Sokolov, A. A. Starobinskii, Dokl. Akad. Nauk SSSR 234, 1043 (Sov. Phys. - Dokl. 22, 312) (1977).

[11] A. A. Abrikosov, Zh. Eksp. Teor. Fiz. 32, 1442 (JETP 5, 1174) (1957).

[12] H. B. Nielsen, P. Olesen, Nucl. Phys. B 61, 45 (1973).

[13] T. W. B. Kibble, Phys. Rep. 67, 183 (1980).

[14] A. Vilenkin, Phys. Rev. D 23, 852 (1981).

[15] R. A. Battye, B. Garbrecht, A. Moss, H. Stoica, J. Cosmol. Astropart. Phys. JCAP 0801, 020 (2008).

[16] Yu. A. Sitenko, N. D. Vlasii, Nucl. Phys. B 787, 241 (2007).

[17] Yu. A. Sitenko, N. D. Vlasii, J. Phys. A: Math. Theor. 41, 164034 (2008). 
[18] G.'t Hooft, Commun. Math. Phys. 117, 685 (1988).

[19] S. Deser, R. Jackiw, Commun. Math. Phys. 118, 495 (1988).

[20] P. de Sousa Gerbert, R. Jackiw, Commun. Math. Phys. 124, 229 (1989).

[21] Yu. A. Sitenko, Nucl. Phys. B 372, 622 (1992).

[22] Yu. A. Sitenko, A. V. Mishchenko, Zh. Eksp. Theor. Fiz. 108, 1516 (JETP 81, 831) (1995).

[23] Yu. A. Sitenko, N. D. Vlasii, in: Astrophysics and Cosmology after Gamow, edited by G. S. Bisnovaty-Kogan et al (Cambridge Scient. Publ., Cambridge UK, 2007) pp. 303-308.

[24] Yu. A. Sitenko, N. D. Vlasii, J. Phys. A: Math. Theor. 43, 354014 (2010).

[25] E. McCann, V. I. Fal'ko, Phys. Rev. Lett. 96, 086805 (2006). 